\title{
PLASMA DIAGNOSTICS FOR THE DIII-D DIVERTOR UPGRADE
}

by

D.N. Hill*, D. Buchenauer, $\dagger$ R. Doerner, $\S$ A. Futch,* C.C. Klepper, $\ddagger$ R. Lehmer, $\S$ B. Leikind, S. Lippmann, M.A. Mahdavi, M. Menon, $\ddagger$ J. Salmonson,,$M$. Schaffer

L. Schmitz, $\S$ J. Smith, and J. Watkins

* Lawrerice Livermore National Laboratory

† Sandia National Laboratory, Livermore

$\ddagger$ Oak Ridge National Laboratory

$\S$ University of California, Los Angeles

"Sandia National Laboratory, Albuquerque

This is a preprint of a paper to be presented at the 8th Topical Conference on High Temperature Plasma Diagnostics, May 7-10, 1990, Hyannis, Massachusetts, and to be printed in the Proceedings.

Work supported by

Department of Energy

Contract DE-AC03-89ER51114

\section{GENERAL ATIOMICS PROJECT 3466 \\ OCTOBER 1990}

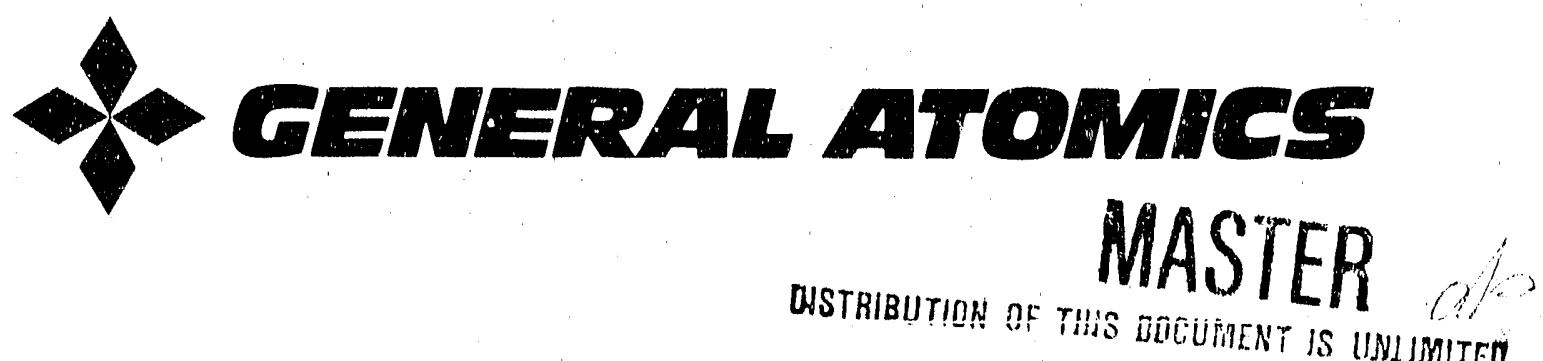




\section{Plasma diagnostics for the DIII-D divertor upgrade}

D.N. Hill, ${ }^{*}$ D. Buchenauer, ${ }^{\dagger}$ R. Doerner,$\S$ A. Fu'TCh, ${ }^{*}$

C.C. Klepper, ${ }^{\ddagger}$ R. LeHMER, ${ }^{\S}$ B. Leikind, S. LipPMANN,

M.A. Mahdavi, M. Menon,${ }^{\ddagger}$ J. Salmonson,, M. Schaffer

L. Schmitz, $\S^{\S}$ J. Smith, AND J. Watkins ${ }^{\sharp}$

General Atomics, San Diego, California 92186-9084

"Lawrence Livermore National Laboratory

$\dagger$ Sandia National Laboratory, Livermore

$\ddagger$ Oak Ridge National Laboratory

$\S$ University of California, Los Angeles

\#Sandia National Laboratory, Albuquerque

The DIII-D tokamak is being upgraded to allow for divertor biasing, baffling, and pumping experiments. This paper gives $2 n$ overview of the new diagnostics added to DIII-D as part of this Advanced Divertor Program. They include tile current monitors, fast reciprocatng Langmuir probes, a fixed probe array in the divertor, fast neutral pressure gauges, and $\mathrm{H}_{\alpha}$ 'leasurements with TV cameras and fiber optics coupled to a high resolution spectrometer. 


\section{INTRODUCTION}

This paper describes the diagnostics added to the DIII-D tokamak as part of the Advanced Divertor Program (ADP). The primary objective of this collaborative program is to test divertor baffling, pumping, and biasing as means of achieving density control in $\mathrm{H}$-mode tokamak plasmas, where it is observed ${ }^{1,2}$, that, even in the absence of external fueling, the density rises to a value proportional to the discharge current. This density rise results from a change in particle confinement and is sustained by neutral recycling in the divertor. Divertor baffling and pumping are predicted ${ }^{3}$ to reduce the core plasma fueling by inhibiting the flow of neutrals out of the divertor region. We expect that cryopumping in the high pressure $\left(10^{-3}\right.$ torr $)$ region under the baffle will be able to remove particles at a rate matching the total fueling from neutral beams (20 torr $-1 / \mathrm{sec})$.

Density control might also be achieved by biasing the outboard divertor target relative to the vacuum vessel so as to drive poloidal currents in the scrape-off-layer (SOL) plasma, as shown in Fig. 1. The poloidal electric field produced by the bias should change the particle and energy flux across the separatrix due to $\mathrm{E} \times \mathrm{B}$ drifts. ${ }^{4}$ In the configuration shown, a positive bias of less than $200 \mathrm{~V}$ on the outermost target is expected to give a net outflux of particles from the core sufficient to cancel the usual density rise observed following the $\mathrm{H}$-mode transition. This voltage is much less than full output of the bias power supply (1200 V and $20 \mathrm{kA}$ ).

The new divertor hardware consists of the three major components indicated in Fig. 2. The biased ring (A) is protected by graphite tiles and serves to define the entrance to the pumping chamber, enclosed by the fully toroidal Inconel baffle (B). 


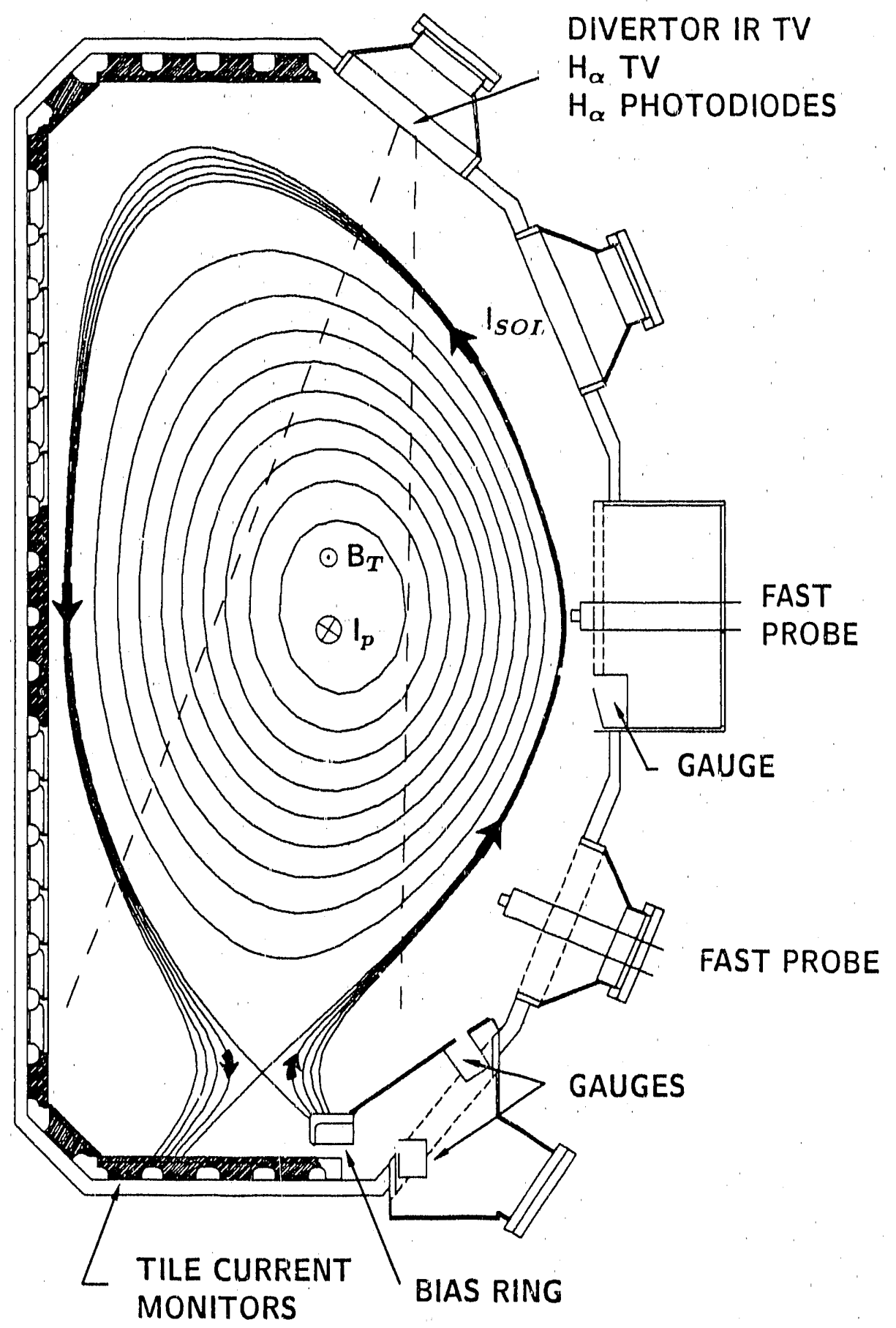

Fig. 1. Cross section of the DIII-D tokamak showing the plasma contacting the divertor bias ring: arrows indicate direction of SOL current flow for positive bias on ring. Direction of plasma current, $I_{p}$, and toroidal field, $\mathrm{B}_{\mathrm{T}}$ are shown. Tiles with current monitors are shaded. 


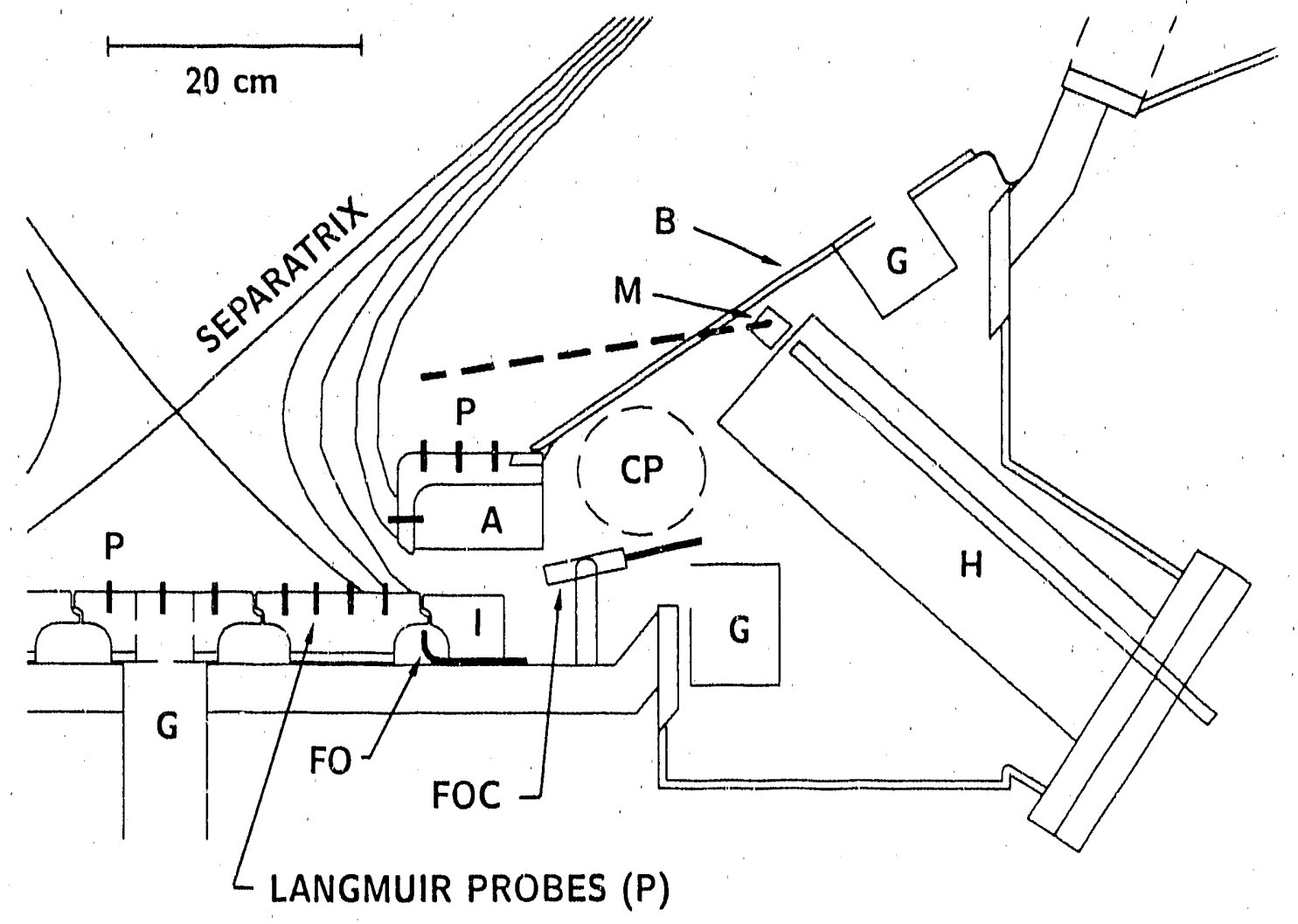

Fig. 2. Detail of the divertor region showing bias ring $(A)$, baffle $(B)$, cryopump and heat shield cross section (CP), fiber optics under the tiles (FO), fiber optics collimator (FOC), pressure gauges ( $G$ ), $H_{\alpha}$ borescope $(H)$, insulators $(I), H_{\alpha}$ viewing mirror $(M)$, and tilemounted Langmuir probes $(P)$. The plasma is positioned for baffling and pumping experiments.

The cryopump cross section is shown schematically (CP). The biased ring is insulated from the vessel by ceramic materials, and is flanked on each side by boron nitride tile rings (I), which establish $1 \mathrm{~cm}$ or more of cross field magnetic insulation. Biasing operation is obtained by positioning the $\mathrm{X}$-point such that the outer leg of the separatrix strikes the bias ring. Pumping will be accomplished by shifting the $\mathrm{X}$-point inward so that the separatrix intercept lies a few $\mathrm{cm}$ (one SOL e-folding length) inside of the lip of the bias ring; this maximizes the flux of neutrals into the pumping chamber. 
Shifting the strike point still further inboard should eliminate plasma interaction with the new hardware entirely.

We have added or upgraded a number of edge plasma diagnostics to help interpret the experimental results. The central diagnostic task for divertor biasing will be to determine the path of the current flow in the SOL plasma and to document how it affects transport. For the pumping and baffling experiments, we must determine the particle throughput to the pumps, as well as the distribution of gas in the plasma boundary. We will briefly describe these diagnostic systems in the remainder of this paper. Section I covers diagnostics for current flow: I-V monitors for the biased ring and an array of tile current monitors. Section II describes how we will diagnose changes in the edge plasma using an array of divertor Langmuir probes and two reciprocating Langmuir probes which can penetrate past the separatrix at the plasma midplane. Section III covers the diagnostics for neutral particles: fast responding pressure gauges and divertor $\mathrm{H}_{\alpha}$ measurements using fiber optics and a tangential-viewing CCD TV camera.

\section{CURRENT MEASUREMENTS}

The purpose of making current measurements is to verify that the biased ring current is flowing in the intended path along field lines in the scrape-off-layer. Ring bias voltage and current will be measured exterior to the vacuum vessel at each of four feed points, which are spaced toroidally at approximately $90^{\circ}$ intervals. Voltage dividers and Hall effect current monitors will be used, respectively. Four feedpoints are used in order to minimize the toroidal current flow in the ring. Localized arcing to 
the vessel should be identifiable from a sudden drop in voltage and a ramping current (the power supply circuit includes disruption-isolating inductors). These monitors may also provide some information on the toroidal symmetry of the SOL current flow, which could be affected by non-axisymmetric plasma contact with the ring.

A more detailed picture of current-flow patterns will be obtained by monitoring the net electrical current flowing to a set of 30 instrumented DIII-D graphite armor tiles. Sixteen of these tiles will be distributed poloidally over the inside half of the vessel, frow the lower divertor up the centerpost to the upper divertor, at a single toroidal position $\left(\theta=202.5^{\circ}\right)$, as indicated by the shading in Fig. 1. Present plans call for the remaining 14 instrumented tiles to be installed in the lower divertor and centerpost at toroidal locations approximately $\pm 120^{\circ}$ from the poloidal array. The base of each instrumented tile is electrically isolated from the vacuum vessel by a thin $(0.025 \mathrm{~mm})$ sheet of mica material that still allows adequate heat conduction to the vessel walls. A path for current flow from the tile to the vessel (at ground potential) is provided by a $2 \mathrm{~m} \Omega$ current shunt resistor which is clamped in place with each tile; the resulting signal is carried outside the vessel via a stainless steel jacketed triaxial cable. Individual tile currents in the range 1-2500 A can be measured.

We have so far tested a prototype array of six tile current monitors in the lower divertor region of DIII-D, and found that they worked as expected. Under normal operating conditions in lower single null discharges similar in shape to that of Fig. I (but without the bias ring in place), we observe scrape-off-layer currents of a few $\mathrm{kA}$ flowing around from the outer divertor strike point to the inner strike point. The net current to the divertor is nearly zero. 


\section{EDGE PLASMA MEASUREMENTS WITH LANGMUIR PROBES}

Changes in the SOL plasma associated with divertor pumping and biasing will be measured with several sets of Langmuir probes: two fast-insertion probes near the plasma midplane (Fig. 1), and an array of 27 single-tip probes located in the divertor tiles and on the biased ring, a subset of which appear in Fig. 2.

The fast reciprocating probes are designed to make a rapid inward plunge into the SOL to measure the plasma parameters up to and even inside of the magnetic separatrix during beam heated $\mathrm{H}$-mode discharges. In $\mathrm{H}$-mode, the plasma density and temperature rise very rapidly inside the separatrix $\left(5 \times 10^{19} \mathrm{~m}^{-3}, 200 \mathrm{eV} \simeq 2 \mathrm{~cm}\right.$

inside for $\left.P_{\mathrm{in}} \simeq 7 \mathrm{MW}\right)$; this produces extremely high thermal loading $\left(\simeq 15 \mathrm{~kW} / \mathrm{cm}^{2}\right)$ and so dwell times must be less than $10 \mathrm{~ms}$. This is achieved using a two stage airdriven insertion mechanism. At the start of a plasma discharge, the first stage slowly moves the fast plunge hardware and an outer shield tube ( $73 \mathrm{~mm}$ diam. Inconel 600) out from the limiter radius to within $10 \mathrm{~cm}$ of the final desired insertion radius. Then, at two desired times, a fast-acting reversible air cylinder forces the inner probe shaft ( $25 \mathrm{~mm}$ diam. Inconel 600) the remaining $10 \mathrm{~cm}$ inward at rates of up to $3 \mathrm{~m} / \mathrm{s}$. Peak deceleration at the inward limit can be as high as $150 \mathrm{G}$. Both the inner probe shaft and outer shield tube have high electrical resistance to ground to reduce the probability of arcing to the vessel walls during a disrupticn. If necessary, the effective dwell time can be reduced below $10 \mathrm{~ms}$ by pushing the plasma away from the probe after the measurement. (The separatrix at the DIII-D outer midplane can be moved at the rate of $1 \mathrm{~cm} / \mathrm{ms}$.) 
The end of the probe shaft is covered by graphite armor, and contains five probe tips (1.5 mm diam. $\times 2 \mathrm{~mm}$ long) made of Graphonal isotropic graphite. In the usual operating mode, two of the tips will measure fluctuations in the plasma floating potential, $\Phi_{f}\left(\mathrm{k}_{\perp}, \omega\right)$, over the frequency and wavenumber range $2 \mathrm{kHz}$ to $1 \mathrm{MHz}$ and 0.1 to $7.5 \mathrm{~cm}^{-1}$. The third tip will $\mathrm{r}$, biased into ion saturation to record density fluctuations, while the remaining two tips will be biased to measure doubleprobe I-V characteristics from which $n_{e}$ and $T_{e}$ profiles can be obtained during the inward plunge. The double probe voltage sweep will be provided by a $10 \mathrm{~kW}$ audio amplifier (TECHRON model 7780) capable of a $1 \mathrm{kV}$ output swing (several times the peak electron temperature just inside the separatrix).

The divertor Langmuir probe array consists of 16 single tip probes mounted in the divertor tiles, plus seven tips mounted on the bias ring. The POCO graphite tips ( $6 \mathrm{~mm}$ diam.) are similar to the JET design ${ }^{5}$, and have rounded ends protruding $1 \mathrm{~mm}$ above the surrounding tile. This minimizes the sensitivity of the projected probe area to changes in the very shallow angle of the field lines $\left(\sim 1-2^{\circ}\right.$ from horizontal $)$ relative to the tiles. The probes can be biased up to $\pm 200 \mathrm{~V}$ and $\pm 4 \mathrm{~A}$; typically, complete I-V characteristics are obtained every $20 \mathrm{~ms}$ throughout the $5 \mathrm{~s}$ discharge. We plan to use a local computer to acquire and process the raw data ( 1 Mbyte) before sending $\mathrm{n}_{e}(r, t)$ and $\mathrm{T}_{e}(r, t)$ values to the main DIII-D acquisition computer.

We have so far operated two prototype probes located at the outer divertor targets and obtained electron density and temperature profiles for ohmic, L-mode, and $\mathrm{H}$-mode plasmas ${ }^{\theta}$. Since the radial spacing of the probes $(3 \mathrm{~cm})$ is relatively large compared to the FWHM of the divertor heat and particle flux profiles $(4 \mathrm{~cm}$ 
or less in $\mathrm{H}$-mode), we had to rely on sweeping the separatrix intercept across the plasma to make detailed profile measurements.

\section{NEUTRAL PARTICLE MEASUREMENTS IN THE DIVERTOR}

The effect of divertor baffing and pumping on global recycling will be determined from extensive neutral pressure and $\mathrm{H}_{\alpha}$ measurements. The resulting data will be interpreted in the context of neutral transport calculations using the DEGAS code, which computes local charge exchange, ionization, and pumping given a known particle source, a fixed plasma background, and details of the divertor geometry. A detailed discussion of the DEGAS modeling can be found in Ref. 7.

The variation of neutral pressure around the plasma will be measured by a set of eight ionization gauges. Two types will be used: magnetically shielded standard Bayard-Alpert gauges mounted outside the toroidal field coils on connecting tubes, and fast-responding ( $<4 \mathrm{~ms}$ ) ionization gauges of the ASDEX type ${ }^{8}$ mounted inside the vacuum vessel at the locations indicated by $(G)$ in Fig. 2. The gauge below the $\mathrm{X}$-point measure the pressure through a $10 \mathrm{~cm}$ hole in the divertor tiles. These ASDEX gauges are mounted within baffled enclosures (about $4 \mathrm{~cm}$ on a side) to force fast atoms to make seyeral bounces before reaching the grid structure. Thus, they measure the wall-temperature molecular neutral pressure, which facilitates comparison with DEGAS results.

The gauges under the baffle plate (a set of two gauges at two toroidal locations) will measure the pressure at the cryopump entrance aperture so that the time-resolved particle removal rate, $\mathrm{Q}$, can be deternined according to $\mathrm{Q}=\mathrm{SP}$. The pumping speed, 
$\mathrm{S}$, of the cryopump will be determined independently using calibrated gas puffs into the torus. In addition, these gauges might also provide some information about the relative atomic/molecular flux into the pump because each has a different entrance-slot orientation: at each toroidal location one faces radially inward towards the entrance under the bias ring (more incident fast atoms) and the other faces $90^{\circ}$ away from it (few atoms, mainly molecules). A fifth gauge is positioned to monitor the pressure just above the baffle which DEGAS simulation suggests is sensitive to the amount of plasma recycling from the bias ring.

Measurements of the divertor $\mathrm{H}_{\alpha}$ emission complement the neutral pressure measurements and provide an important benchmark for the neutral transport modeling. Two-dimensional tangential views of the divertor region will be provided by a CCD TV camera system which has been modified to accommodate installation of the bias ring and baffle. The tangential view is obtained using an Olympus borescope fitted inside a reentrant tube mounted on the port under the baffle (Fig. 2). The tube terminates under the baffle for protection against plasma disruptions and so a cutout in the baffle (sealed against gas leakage) with a metal mirror (M) is required to achieve the correct field of view. Images from the CCD camera (EEV P45380/V1, chosen for its relative insensitivity to neutron damage) are stored on video tape for later digitization and analysis.

We will also make spectrally resolved $\mathrm{H}_{\alpha}$ measurements at the recycling zone under the bias ring using vacuum-compatible fiber optics ${ }^{\theta}$. Two of the fibers will be connected to small single lens collimators (labeled FOC in Fig. 2) to give a radial and a toroidal view. Two additional fibers are being mounted under the outermost row 
of divertor tiles and will look upwards in the gap between tiles to view the divertor plasma at the outer strike point. The output from the fibers is coupled to a grating spectrometer and OMA so that the hydrogen line shapes (and thus atomic velocities) can be studied in detail $(0.08 \AA$ resolution $)$. Use of a lower dispersion grating will also allow the system to be used for general surveys of impurity-line emission.

\section{SUMMARY}

In this paper we have given a brief overview of the new diagnostics being added to the DIII-D tokamak as part of the Advanced Divertor Program. These diagnostics will be used to quantify how divertor biasing, baftling, and pumping modify the scrape-off-layer plasma. Divertor Langmuir probes and tile current monitors will heip determine the magnitude and path of the edge currents produced by biasing. The fast reciprocating Langmuir probes will be used to measure changes in the SOL plasma transport. And the $\mathrm{H}_{\alpha}$ and neutral pressure measurements will, in conjunction with neutral transport modeling, help quantify the effect that baffling and pumping will have on plasma fueling by neutral recycling in the divertor. These measurements will help us understand the global effects produced by the new divertor geometry, given the complex coupling between the edge and core plasmas.

\section{ACKNOWLEDGEMENTS}

The authors wish to thank P. Anderson, R. Callis, J. Luxon, P. Petersen, and R. Stambaugh of General Atomics for their helpful review of these diagnostics. We would also like to thank G. Matthews of Culham Laboratories for useful 
suggesti ns on the design of the Langmuir probes and on the use of the EEV cameras. This vork is supported by the U.S. Department of Energy under Contract Nos. DE-AC03-76DP00789, DE-ACú-84OR21400, DE-AC03-89ER51114, DE-FG0389ER51121, and W-7405-ENG-48. 


\section{References}

1 ASDEX Team, Nucl. Fusion 29, 1959 (1989).

2 D. P. Schissel, K. H. Burrell, J. C. DeBoo, et al., Nucl. Fusion 29, 185 (1989).

3 M. A. Mahdavi, M. Schaffer, P. Mioduszewski, et al., General Atomics Report GA-A19547, February 1989.

4 G. M. Staebler and F. L. Hinton, Nucl. Fusion 29, 1820 (1989).

5 P.J. Harbour, J. Nucl. Mater. 162-164, (1989).

6 D. Buchenauer; W.L. Hsu, and D.N. Hill, these proceedings.

7 S. L. Allen, M. E. Rensink, D. N. Hill, et al., J. Nucl. Mater. 162-164, 80 (1.989).

${ }^{8}$ G. L. Jackson, T. E. Evans, M. A. Mahdavi, and G. I'n zs, Bull. Am. Phys. Soc. (1987).

${ }^{9}$ C.C. Klepper, J. Simkins, D.L. Hillis, et al., these proceedings. 
